\title{
Experimental and finite element studies on free vibration of skew plates
}

\author{
C. V. Srinivasa $\cdot$ Y. J. Suresh · W. P. Prema Kumar
}

Received: 17 September 2013/Accepted: 27 December 2013/Published online: 21 February 2014

(C) The Author(s) 2014. This article is published with open access at Springerlink.com

\begin{abstract}
The present paper deals with the experimental and finite element studies carried out on free vibration of isotropic and laminated composite skew plates. The natural frequencies were determined using CQUAD8 finite element of MSC/NASTRAN and comparison made between the experimental values and the finite element solution. The effects of the skew angle and aspect ratio on the natural frequencies of isotropic skew plates were studied. The effects of skew angle, aspect ratio, fiber orientation angle and laminate stacking sequence (keeping total number of layers in the laminate constant) on the natural frequencies of antisymmetric laminated composite skew plates were also studied. The experimental values of the natural frequencies are in good agreement with the finite element solution. The natural frequencies generally increase with an increase in the skew angle for any given value of aspect ratio.
\end{abstract}

Keywords Skew plate $\cdot$ Natural frequency $\cdot$ Aspect ratio . Fibre orientation angle $\cdot$ Finite element analysis

C. V. Srinivasa $(\bowtie)$

Department of Mechanical Engineering, GM Institute of

Technology, Davangere 577006, Karnataka, India

e-mail: srinivas@gmit.info

\section{Y. J. Suresh}

Department of Mechanical Engineering, J.N.N. College of

Engineering, Shivamogga 577204, Karnataka, India

e-mail: yjsuresh06@gmail.com

\section{W. P. Prema Kumar}

Department of Civil Engineering, Reva Institute of Technology

and Management, Reva University,

Bangalore 560064, Karnataka, India

e-mail: wppremakumar@yahoo.co.in

\section{List of symbols}

a Plate length

$b \quad$ Plate width

$t \quad$ Plate thickness

$N L \quad$ Number of layers in the laminate

$E$ Modulus of elasticity of the material of isotropic plate

$E_{1} \quad$ Young's modulus of the lamina in the longitudinal direction

$E_{2} \quad$ Young's modulus of the lamina in the transverse direction

$G_{12}$ In-plane shear modulus of the lamina

$\alpha \quad$ Skew angle of the plate

$\theta \quad$ Fiber orientation angle of the lamina

$\mu \quad$ Poisson's ratio of the material of isotropic plate

$v_{12} \quad$ Major Poisson's ratio of the lamina

$\rho \quad$ Mass density of the material of the plate

$\rho_{1} \quad$ Mass density per unit area

$D \quad$ Flexural rigidity of isotropic plate, $E t^{3} / 12\left(1-\mu^{2}\right)$

$\omega \quad$ Natural angular frequency of plate

$K_{\mathrm{f}} \quad$ Non-dimensional frequency coefficient

\section{Introduction}

The skew plates have wide application in civil, marine, aeronautical and mechanical engineering, some of them being wings of aeroplanes, skew bridges, ship hulls and parallelogram slabs in buildings. The exact solutions to free vibration problems of skew plates are mathematically involved and in many complicated cases not available. Hence, most of the available solutions are based on approximate methods, the most commonly used method being the finite element analysis. 
The earlier studies on free vibration characteristics of skew plates are those of Barton (1951), Kaul and Cadambe (1956) and Hasegawa (1957) using Rayleigh-Ritz method. Hamada (1959) applied the Lagrangian multiplier technique to obtain the fundamental frequency of rhombic skew plate. Classen (1963) extended the work of Barton (1951) by adopting a Fourier sine series solution scheme in conjunction with the Rayleigh-Ritz method. Conway and Farnham (1965) employed the point matching method to study the free vibration of triangular, rhombic and parallelogram plates. The frequencies were calculated for different values of skew angle for both simply supported and clamped boundary conditions. Laura and Grosson (1968) obtained fundamental frequencies of vibration for simply supported rhombic plates using conformal mapping and Galerkin's method and compared their results with those of Conway and Farnham (1965). The discrepancy has been observed to increase with the skew angle.

Monforton (1968) obtained the fundamental frequencies of clamped rhombic plates using finite element method. The frequencies and mode shapes of clamped skew plates were studied by Durvasula (1969) using Galerkin's method. The deflection function was expressed as a double series of beam characteristic functions in terms of skew coordinates to satisfy the zero deflection and normal slope on all the edges of the plate. The results obtained were compared with the results of Kaul and Cadambe (1956), Hasegawa (1957) and Conway and Farnham (1965). Thangam Babu and Reddy (1971) investigated the free vibration characteristics of orthotropic skew plates with two opposite edges simply supported and the other two edges free. Nair and Durvasula (1973) reported the natural frequencies of isotropic and orthotropic skew plates for simply supported, clamped, free edge boundary conditions and also for combinations of these conditions. Srinivasan and Ramachandran (1975) employed a numerical method to obtain the natural frequencies and mode shapes of orthotropic skew plates. Kuttler and Sigillito (1980) used trial function method to solve the free vibration problem of skew plates.

Mizusuwa et al. (1979, 1980) and Mizusuwa and Kajita (1986, 1987) employed the Rayleigh-Ritz method with B-spline functions and studied the effects of skew angle and location of point supports on natural frequencies of isotropic skew plates. Liew and Lam (1990) used a set of 2-D orthogonal plate functions as admissible deflection functions and studied the flexural vibration of skew plates using Rayleigh-Ritz method. They obtained the free vibration characteristics of rhombic plates with different boundary conditions. Bardell (1992) adopted the hierarchical finite element method to determine the natural frequencies and mode shapes of isotropic skew plates. Liew and Wang (1993) employed the Rayleigh-Ritz method and obtained the results for rhombic plates with different boundary conditions by varying the location of internal support, skew angle and aspect ratio. Singh and Chakraverthy (1994) evaluated the first five frequencies of the transverse vibration of skew plates under different boundary conditions using orthogonal polynomials. McGee and Butalia (1994) studied the free vibration of thick and thin cantilever skew plates using $C^{0}$ continuous isoparametric quadrilateral element.

Kamal and Durvasula (1986) studied the free vibration characteristics of laminated composite plates using modified shear deformation layered composite theory and Rayleigh-Ritz energy approach. Malhotra et al. (1988) studied the free vibration characteristics of rhombic orthotropic plates using a parallelogram orthotropic plate finite element for various boundary conditions and skew angles. Krishnan and Deshpande (1992) employed DKT finite element to determine the effects of fiber orientation angle, skew angle, aspect ratio and length-to-thickness ratio on the fundamental frequencies of single layer Graphite/Epoxy and Glass/Epoxy skew plates.

Krishna Reedy and Palaninathan (1999) used a high precision triangular plate bending element to study the free vibration characteristics of laminated composite skew plates. A consistent mass matrix has been used in the study. Singha and Ganapathi (2004) studied the free flexural vibration characteristics of laminated composite skew plates using finite element approach. Garg et al. (2006) have carried out free vibration studies on isotropic, orthotropic, and layered anisotropic composite and sandwich skew plates using isoparametric finite element model.

Clary (1975) investigated theoretically and experimentally the effect of fiber orientation on the first five flexural modes of free vibration of rectangular unidirectional Boron/Epoxy panels. The theoretical and experimental values of frequencies were in good agreement except in the case of thinner panels where there was appreciable error. Cawley and Adams (1978) used finite element method which included the effect of transverse shear deformation to predict the natural modes of free-free CFRP plates. Maruyama et al. (1983) used the real-time technique of holographic interferometry to determine the natural frequencies and the corresponding mode shapes of transverse vibration of clamped trapezoidal plates. Chakraborty et al. (2000) made studies on free vibration response of FRP composite plates using experimental and numerical techniques. Dutt and Shivanand (2011) studied the free vibration response of woven carbon composite laminated plates with $\mathrm{C}-\mathrm{F}-\mathrm{F}-\mathrm{F}$ (one edge clamped and the other three free) and $\mathrm{C}-\mathrm{F}-\mathrm{C}-\mathrm{F}$ (two opposite edges clamped and the other two edges free) boundary conditions using a FFT analyzer and compared the results obtained with finite element solution. Today the skew plate problem is widely 
recognized as a benchmark in the testing of a newly developed finite element. Very few experimental studies have been made on laminated composite skew plates and the finite element solution experimentally validated. The present paper is an attempt to address this aspect at least in a partial manner.

\section{Test specimens and experimental set-up}

\section{Test specimens}

In this study, isotropic plates made of Aluminum 7075-T6 were used. The material was supplied by the Rio-Tinto Alcon, Canada (Pechiney Aluminum, France). The laminated composite plate specimens were fabricated by hand lay-up technique using unidirectional Glass fibers, Epoxy556 Resin and the Hardener (HY951) supplied by CibaGeigy India Ltd. The fiber weight percentage is 50:50. The test specimens were prepared in accordance with the relevant ASTM provisions. The material properties of the isotropic plates made of Aluminum 7075-T6 are: $E=71.7 \mathrm{GPa}, \mu=0.33$ and $\rho=2,800 \mathrm{~kg} / \mathrm{m}^{3}$ and these were supplied by the manufacturer Rio-Tinto Alcon, Canada. For laminated Glass/Epoxy composite plate, the material constants $E_{1}$ and $E_{2}$ were evaluated experimentally using Instron Universal Testing Machine as per ASTM Standard D 3039/D 3039 M (2006). The average of three experimental determinations was adopted. For the determination of Poisson's ratio $v_{12}$, two strain gages were bonded to the specimen, one in the direction of the loading and the other at right angles to it. The strains were measured in longitudinal and transverse directions using strain indicator. The ratio of transverse to longitudinal strain gives the Poisson's ratio within the elastic range. The average of three experimental determinations was adopted. The shear modulus $G_{12}$ was computed using standard expression available in Jones (1975). The adopted material properties are: $E_{1}=38.07 \mathrm{GPa}, \quad E_{2}=8.1 \mathrm{GPa}$, $\mathrm{G}_{12}=3.05 \mathrm{GPa}, v_{12}=0.22, \rho=2,200 \mathrm{~kg} / \mathrm{m}^{3}$. The aspect ratio of the test specimens was varied from 1.0 to 2.5 .

\section{Experimental set-up}

The experimental set-up is shown in Fig. 1. First, the test specimen was placed in the fixture shown in Fig. 1 with two opposite edges fully clamped and the remaining two edges completely free. The piezoelectric accelerometer was directly mounted on the test specimen at the geometric center using an adhesive. The accelerometer was then connected to signal-conditioning unit (Fast Fourier Transform Analyzer), where the signal goes through the charge amplifier and an Analog-to-Digital Converter. The plate

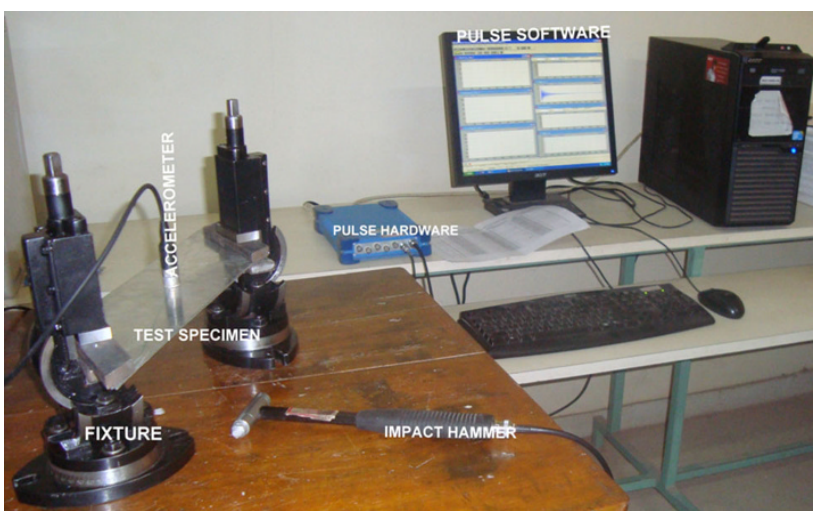

Fig. 1 Experimental Set-up

was excited in a selected point by means of impact hammer. The impact hammer was struck at the selected point five times and the average value of the frequency response function (FRF) was input to the computer through an USB port. Sufficient precautions were taken for ensuring that the strike of the impact hammer was normal to the surface of the plate. The pulse lab software accompanying the equipment was used for recording the signals directly in the memory of the computer. The signal was then read and processed to extract different features including frequencies. The frequencies were measured by moving the cursor to the peaks of the FRF. Five separate experimental determinations were done for the natural frequency of each specimen and then the average value was adopted.

\section{Finite element solution}

A finite element analysis was made for obtaining the first three natural frequencies using MSC/NASTRAN software. CQUAD8 (eight-noded isoparametric curved shell element) was employed as it yields better results compared to CQUAD4 element of the said software as revealed by the investigation reported by Srinivasa et al. (2012). Several options exist in the software in respect of real eigenvalue extraction, and the Lanczos method was used in the present study as it combines the best features of the other methods and computes accurate eigenvalues and eigenvectors.

\section{Results and discussion}

The results of the present work are presented in tables and figures in terms of non-dimensional frequency coefficient $\left(K_{\mathrm{f}}\right)$ defined by $K_{\mathrm{f}}=\frac{\omega a^{2}}{\pi^{2}} \sqrt{\frac{\rho t}{D}}$ for isotropic plates and by $K_{\mathrm{f}}=\omega a^{2} \sqrt{\frac{\rho_{1}}{E_{2} t^{3}}}$ for laminated composite plates (Srinivasa et al. 2013). 
Table 1 Non-dimensional frequency coefficient for isotropic skew plates

\begin{tabular}{|c|c|c|c|c|c|c|c|c|c|}
\hline \multirow[t]{4}{*}{ Aspect ratio $(a / b)$} & \multirow[t]{4}{*}{ Mode number } & \multicolumn{8}{|c|}{ Non-dimensional frequency coefficient $\left(K_{\mathrm{f}}\right)$} \\
\hline & & \multicolumn{8}{|c|}{ Skew angle $(\alpha)$} \\
\hline & & \multicolumn{2}{|l|}{$0^{\circ}$} & \multicolumn{2}{|l|}{$15^{\circ}$} & \multicolumn{2}{|l|}{$30^{\circ}$} & \multicolumn{2}{|l|}{$45^{\circ}$} \\
\hline & & Experiment & FEM & Experiment & FEM & Experiment & FEM & Experiment & FEM \\
\hline \multirow[t]{3}{*}{1.0} & 1 & $2.978(0.10)$ & 3.102 & $3.216(0.11)$ & 3.265 & $3.732(0.12)$ & 3.822 & $4.946(0.15)$ & 5.066 \\
\hline & 2 & $3.518(0.12)$ & 3.665 & $3.735(0.13)$ & 3.792 & $4.133(0.14)$ & 4.233 & $5.136(0.15)$ & 5.260 \\
\hline & 3 & $5.795(0.15)$ & 6.036 & $6.123(0.16)$ & 6.216 & $6.708(0.17)$ & 6.870 & $8.383(0.18)$ & 8.585 \\
\hline \multirow[t]{3}{*}{1.5} & 1 & $3.028(0.11)$ & 3.080 & $3.185(0.11)$ & 3.241 & $3.548(0.12)$ & 3.711 & $4.833(0.13)$ & 4.761 \\
\hline & 2 & $4.169(0.13)$ & 4.241 & $4.313(0.12)$ & 4.388 & $4.587(0.13)$ & 4.798 & $5.832(0.14)$ & 5.779 \\
\hline & 3 & $8.343(0.17)$ & 8.487 & $8.817(0.18)$ & 8.970 & $9.519(0.19)$ & 9.957 & $11.483(0.20)$ & 11.361 \\
\hline \multirow[t]{3}{*}{2.0} & 1 & $3.001(0.11)$ & 3.071 & $3.151(0.11)$ & 3.206 & $3.538(0.12)$ & 3.602 & $4.342(0.13)$ & 4.418 \\
\hline & 2 & $4.825(0.12)$ & 4.939 & $5.000(0.14)$ & 5.087 & $5.433(0.15)$ & 5.532 & $6.484(0.15)$ & 6.596 \\
\hline & 3 & $8.270(0.16)$ & 8.465 & $8.747(0.15)$ & 8.898 & $9.998(0.16)$ & 10.180 & $12.554(0.19)$ & 12.771 \\
\hline \multirow[t]{3}{*}{2.5} & 1 & $3.006(0.10)$ & 3.064 & $3.130(0.10)$ & 3.181 & $3.417(0.11)$ & 3.512 & $4.052(0.12)$ & 4.150 \\
\hline & 2 & $5.591(0.12)$ & 5.699 & $5.764(0.12)$ & 5.858 & $6.168(0.13)$ & 6.339 & $7.331(0.13)$ & 7.508 \\
\hline & 3 & $8.281(0.14)$ & 8.442 & $8.709(0.14)$ & 8.850 & $9.749(0.15)$ & 10.019 & $11.972(0.16)$ & 12.260 \\
\hline
\end{tabular}

The number in parentheses represents the standard deviation

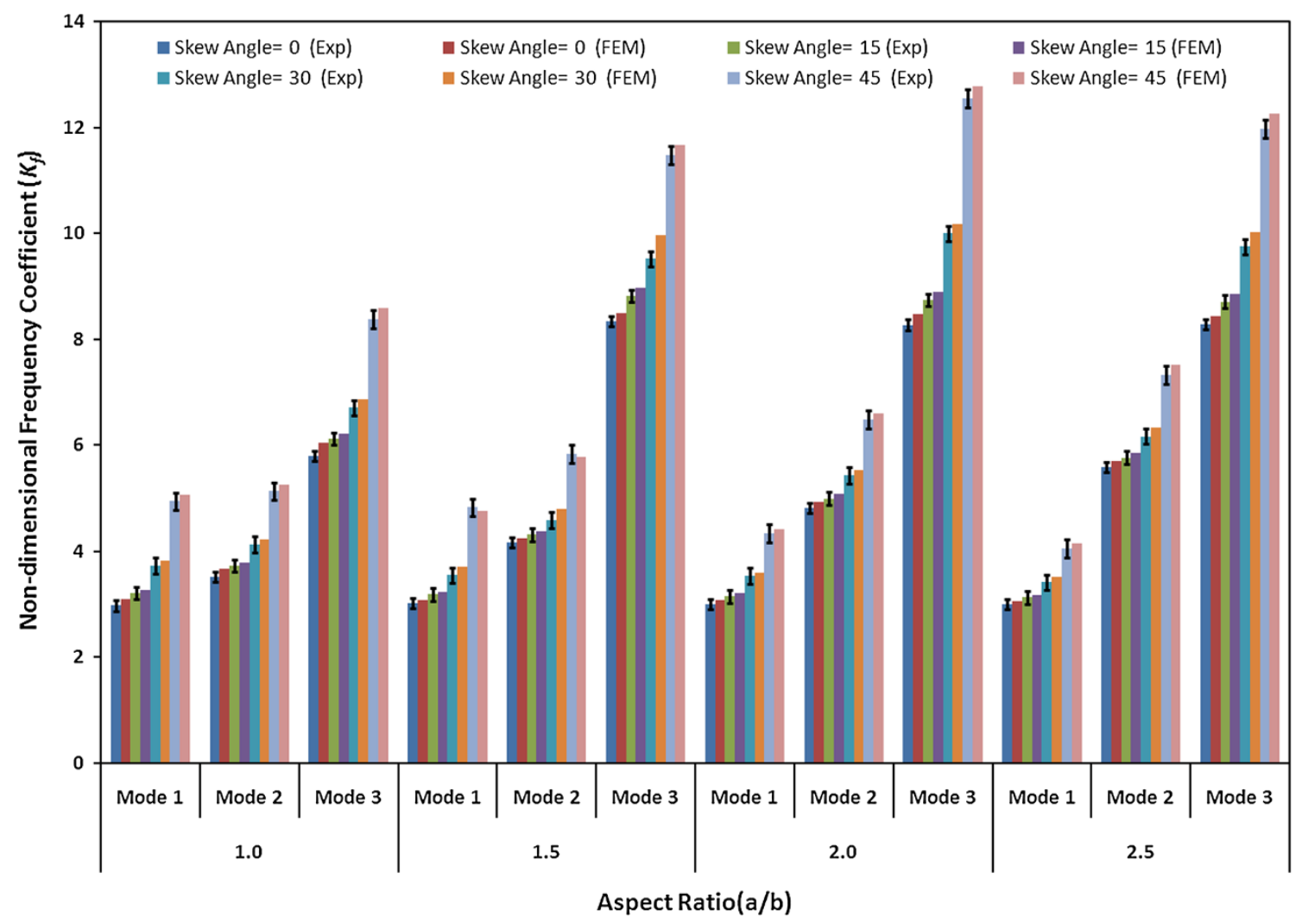

Fig. 2 Variation of $K_{\mathrm{f}}$ with aspect ratio $(a / b)$ for isotropic skew plates 


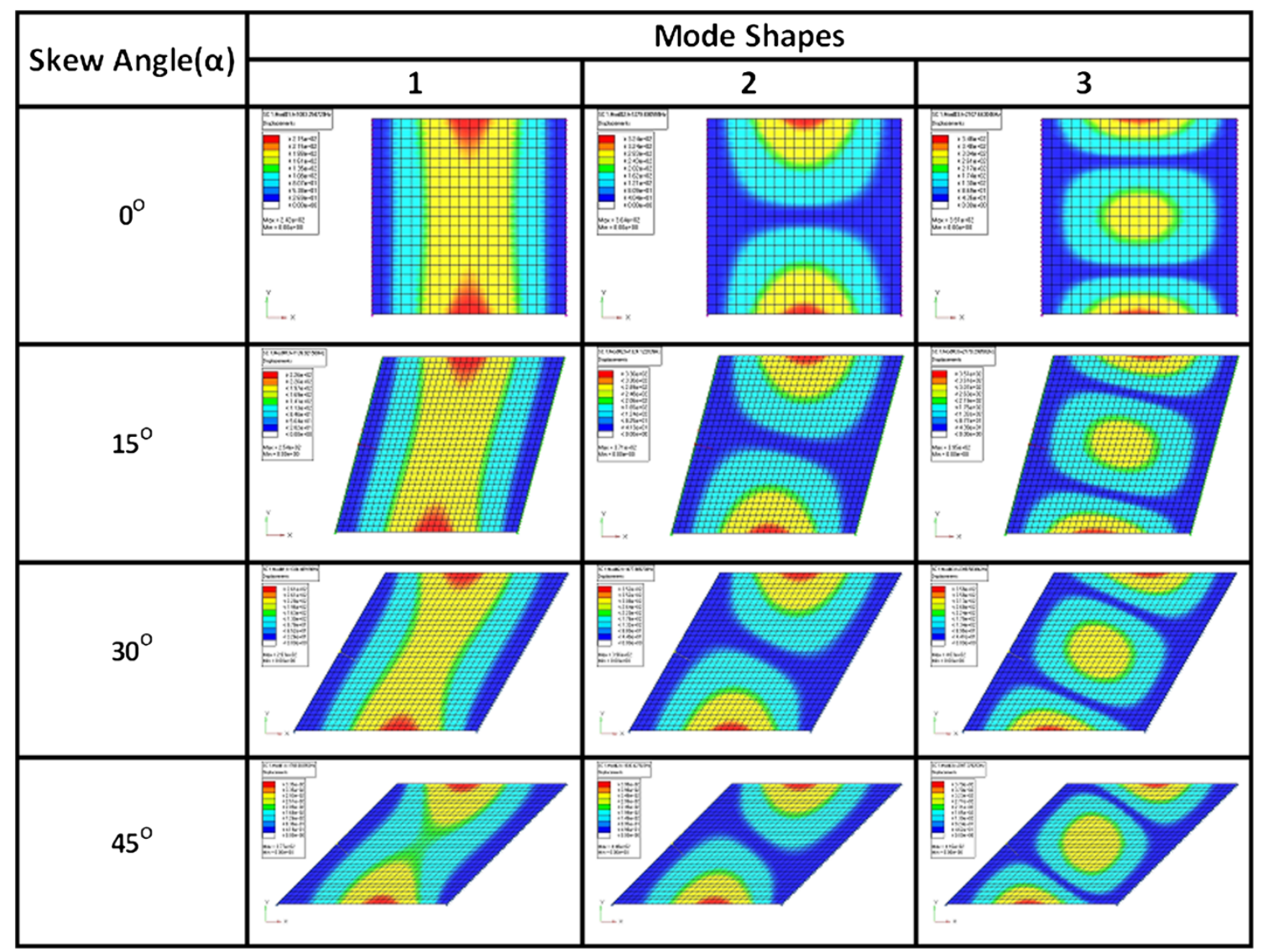

Fig. 3 Mode shapes for isotropic skew plates $(a / b=1.0)$

Table 2 Non-dimensional frequency coefficient for laminated composite skew plates $\left(\alpha=0^{\circ}\right)$

\begin{tabular}{|c|c|c|c|c|c|c|c|c|c|}
\hline \multirow{4}{*}{$\begin{array}{l}\text { Laminate staking } \\
\text { sequence }\end{array}$} & \multirow{4}{*}{$\begin{array}{l}\text { Mode } \\
\text { number }\end{array}$} & \multicolumn{8}{|c|}{ Non-dimensional frequency coefficient $\left(K_{f}\right)$} \\
\hline & & \multicolumn{8}{|c|}{ Aspect ratio $(a / b)$} \\
\hline & & \multicolumn{2}{|l|}{1.0} & \multicolumn{2}{|l|}{1.5} & \multicolumn{2}{|l|}{2.0} & \multicolumn{2}{|l|}{2.5} \\
\hline & & Experiment & FEM & Experiment & FEM & Experiment & FEM & Experiment & FEM \\
\hline \multirow{3}{*}{$\begin{array}{l}\text { Angle-ply } \\
\qquad\left[+0^{\circ} /-0^{\circ} \% / 0^{\circ}\right]\end{array}$} & 1 & $15.304(0.13)$ & 15.617 & $15.180(0.11)$ & 15.649 & $14.983(0.40)$ & 15.607 & $14.824(0.40)$ & 15.604 \\
\hline & 2 & $15.990(0.10)$ & 16.316 & $16.683(0.23)$ & 17.199 & $17.536(0.13)$ & 18.267 & $18.613(0.34)$ & 19.593 \\
\hline & 3 & $19.435(0.12)$ & 19.832 & $26.194(0.15)$ & 27.004 & $36.684(0.90)$ & 38.213 & 40.859 (1.39) & 43.010 \\
\hline \multirow{3}{*}{$\begin{array}{l}\text { Angle-ply } \\
\qquad\left[+45^{\circ} /-45^{\circ} / \ldots /-45^{\circ}\right]\end{array}$} & 1 & $9.454(0.13)$ & 9.647 & $9.260(0.16)$ & 9.547 & $9.039(0.12)$ & 9.416 & $8.855(0.12)$ & 9.321 \\
\hline & 2 & $12.851(0.08)$ & 13.114 & $15.880(0.12)$ & 16.371 & $19.119(0.34)$ & 19.916 & $22.488(0.30)$ & 23.671 \\
\hline & 3 & $22.684(0.34)$ & 23.147 & $25.673(0.12)$ & 26.467 & $25.069(0.45)$ & 26.114 & $24.548(0.90)$ & 25.840 \\
\hline \multirow{3}{*}{$\begin{array}{l}\text { Angle-ply } \\
\qquad\left[+90^{\circ} /-90^{\circ} / \ldots /-90^{\circ}\right]\end{array}$} & 1 & $7.055(0.07)$ & 7.199 & $6.996(0.09)$ & 7.213 & $6.905(0.12)$ & 7.193 & $6.831(0.11)$ & 7.191 \\
\hline & 2 & $8.448(0.18)$ & 8.620 & $9.818(0.08)$ & 10.121 & $11.356(0.22)$ & 11.830 & $13.021(0.29)$ & 13.707 \\
\hline & 3 & $19.399(0.20)$ & 19.795 & $19.283(0.018)$ & 19.879 & $19.031(0.34)$ & 19.824 & $18.827(0.02)$ & 19.818 \\
\hline \multirow{3}{*}{$\begin{array}{l}\text { Cross-ply } \\
{\left[0^{\circ} / 90^{\circ} \% . \ldots 190^{\circ}\right]}\end{array}$} & 1 & $11.901(0.16)$ & 12.144 & $11.805(0.20)$ & 12.170 & $11.654(0.12)$ & 12.139 & $11.530(0.34)$ & 12.137 \\
\hline & 2 & $12.777(0.22)$ & 13.038 & $13.686(0.21)$ & 14.109 & $14.784(0.25)$ & 15.400 & $16.090(0.21)$ & 16.937 \\
\hline & 3 & $19.420(0.25)$ & 19.817 & $32.410(0.30)$ & 33.412 & $32.120(0.33)$ & 33.458 & $31.780(1.25)$ & 33.452 \\
\hline
\end{tabular}

The number in parentheses represents the standard deviation 
Table 3 Non-dimensional frequency coefficient for laminated composite skew plates $\left(\alpha=15^{\circ}\right)$

\begin{tabular}{|c|c|c|c|c|c|c|c|c|c|}
\hline \multirow{4}{*}{$\begin{array}{l}\text { Laminate staking } \\
\text { sequence }\end{array}$} & \multirow{4}{*}{$\begin{array}{l}\text { Mode } \\
\text { number }\end{array}$} & \multicolumn{8}{|c|}{ Non-dimensional frequency coefficient $\left(K_{f}\right)$} \\
\hline & & \multicolumn{8}{|c|}{ Aspect ratio $(a / b)$} \\
\hline & & \multicolumn{2}{|l|}{1.0} & \multicolumn{2}{|l|}{1.5} & \multicolumn{2}{|l|}{2.0} & \multicolumn{2}{|l|}{2.5} \\
\hline & & Experiment & FEM & Experiment & FEM & Experiment & FEM & Experiment & FEM \\
\hline \multirow{3}{*}{$\begin{array}{l}\text { Angle-ply } \\
\qquad\left[+0^{\circ} /-0^{\circ} \% \ldots /-0^{\circ}\right]\end{array}$} & 1 & $15.346(0.05)$ & 15.821 & $15.350(0.20)$ & 15.990 & $14.997(0.30)$ & 15.787 & $14.495(0.45)$ & 15.420 \\
\hline & 2 & $16.103(0.15)$ & 16.602 & $16.502(0.15)$ & 17.190 & $17.739(0.12)$ & 18.672 & $18.852(0.56)$ & 20.056 \\
\hline & 3 & $19.876(0.03)$ & 20.491 & $26.997(0.11)$ & 28.122 & $37.684(0.18)$ & 39.668 & $40.917(1.98)$ & 43.528 \\
\hline \multirow{3}{*}{$\begin{array}{l}\text { Angle-ply } \\
\qquad\left[+45^{\circ} /-45^{\circ} / \ldots /-45^{\circ}\right]\end{array}$} & 1 & $10.235(0.07)$ & 10.552 & $9.688(0.06)$ & 10.092 & $9.498(0.12)$ & 9.998 & $9.253(0.90)$ & 9.844 \\
\hline & 2 & $13.044(0.03)$ & 13.447 & $16.013(0.03)$ & 16.680 & $18.952(0.40)$ & 19.949 & $22.047(1.00)$ & 23.455 \\
\hline & 3 & $22.592(0.07)$ & 23.291 & $26.898(0.08)$ & 28.019 & $27.056(0.35)$ & 28.479 & $25.625(1.08)$ & 27.260 \\
\hline \multirow{3}{*}{$\begin{array}{l}\text { Angle-ply } \\
\qquad\left[+90^{\circ} /-90^{\circ} / \ldots /-90^{\circ}\right]\end{array}$} & 1 & $7.368(0.03)$ & 7.596 & $7.253(0.10)$ & 7.556 & $7.107(0.10)$ & 7.481 & $6.976(0.08)$ & 7.421 \\
\hline & 2 & $8.909(0.11)$ & 9.184 & $10.238(0.11)$ & 10.664 & $11.722(0.20)$ & 12.339 & $13.660(0.45)$ & 14.532 \\
\hline & 3 & $18.957(0.02)$ & 19.544 & $20.157(0.14)$ & 20.996 & $19.726(0.22)$ & 20.765 & $19.583(0.08)$ & 20.833 \\
\hline \multirow{3}{*}{$\begin{array}{l}\text { Cross-ply } \\
{\left[0^{\circ} / 90^{\circ} \% \ldots / 90^{\circ}\right]}\end{array}$} & 1 & $12.032(0.09)$ & 12.404 & $12.099(0.18)$ & 12.603 & $11.740(0.23)$ & 12.358 & $11.480(0.34)$ & 12.212 \\
\hline & 2 & $13.092(0.02)$ & 13.497 & $14.051(0.09)$ & 14.636 & $15.159(0.18)$ & 15.957 & $17.075(0.88)$ & 18.165 \\
\hline & 3 & $20.156(0.11)$ & 20.779 & 31.759 (1.05) & 33.082 & $32.392(1.57)$ & 34.097 & $32.422(1.55)$ & 34.492 \\
\hline
\end{tabular}

The number in parentheses represents the standard deviation

Table 4 Non-dimensional frequency coefficient for laminated composite skew plates $\left(\alpha=30^{\circ}\right)$

\begin{tabular}{|c|c|c|c|c|c|c|c|c|c|}
\hline \multirow{4}{*}{$\begin{array}{l}\text { Laminate staking } \\
\text { sequence }\end{array}$} & \multirow{4}{*}{$\begin{array}{l}\text { Mode } \\
\text { number }\end{array}$} & \multicolumn{8}{|c|}{ Non-dimensional frequency coefficient $\left(K_{f}\right)$} \\
\hline & & \multicolumn{8}{|c|}{ Aspect ratio $(a / b)$} \\
\hline & & \multicolumn{2}{|l|}{1.0} & \multicolumn{2}{|l|}{1.5} & \multicolumn{2}{|l|}{2.0} & \multicolumn{2}{|l|}{2.5} \\
\hline & & Experiment & FEM & Experiment & FEM & Experiment & FEM & Experiment & FEM \\
\hline \multirow{3}{*}{$\begin{array}{l}\text { Angle-ply } \\
\qquad\left[+0^{\circ} /-0^{\circ} \% \ldots /-0^{\circ}\right]\end{array}$} & 1 & $16.014(0.09)$ & 16.595 & $15.812(0.33)$ & 16.557 & $15.543(0.23)$ & 16.448 & $15.314(0.32)$ & 16.379 \\
\hline & 2 & $16.999(0.08)$ & 17.616 & $17.909(0.25)$ & 18.753 & $18.955(0.25)$ & 20.058 & $20.203(0.40)$ & 21.608 \\
\hline & 3 & $21.958(0.35)$ & 22.755 & $30.296(0.85)$ & 31.723 & $41.246(1.24)$ & 43.646 & $42.345(0.90)$ & 45.289 \\
\hline \multirow{3}{*}{$\begin{array}{l}\text { Angle-ply } \\
\qquad\left[+45^{\circ} /-45^{\circ} / \ldots /-45^{\circ}\right]\end{array}$} & 1 & $12.972(0.21)$ & 13.442 & $12.003(0.30)$ & 12.569 & $11.117(0.12)$ & 11.764 & $10.461(0.18)$ & 11.188 \\
\hline & 2 & $14.369(0.25)$ & 14.890 & $16.867(0.50)$ & 17.662 & $19.881(0.45)$ & 21.038 & $22.708(0.20)$ & 24.286 \\
\hline & 3 & $23.764(0.40)$ & 24.626 & $33.427(1.20)$ & 35.002 & $33.081(1.34)$ & 35.006 & $32.189(0.45)$ & 34.426 \\
\hline \multirow{3}{*}{$\begin{array}{l}\text { Angle-ply } \\
\qquad\left[+90^{\circ} /-90^{\circ} / \ldots /-90^{\circ}\right]\end{array}$} & 1 & $8.892(0.15)$ & 9.214 & $8.474(0.21)$ & 8.873 & $8.079(0.15)$ & 8.549 & $7.780(0.08)$ & 8.321 \\
\hline & 2 & $10.655(0.25)$ & 11.042 & $11.900(0.20)$ & 12.461 & $13.312(0.12)$ & 14.087 & $14.902(0.30)$ & 15.938 \\
\hline & 3 & $20.408(0.40)$ & 21.149 & $23.573(0.40)$ & 24.683 & $22.761(0.33)$ & 24.085 & $22.164(0.35)$ & 23.705 \\
\hline \multirow{3}{*}{$\begin{array}{l}\text { Cross-ply } \\
{\left[0^{\circ} / 90^{\circ} \% / . .190^{\circ}\right]}\end{array}$} & 1 & $13.020(0.22)$ & 13.492 & $12.778(0.30)$ & 13.380 & $12.488(0.28)$ & 13.215 & $12.242(0.15)$ & 13.093 \\
\hline & 2 & $14.555(0.20)$ & 15.082 & $15.690(0.35)$ & 16.429 & $16.832(0.30)$ & 17.811 & $18.175(0.24)$ & 19.439 \\
\hline & 3 & $23.111(0.35)$ & 23.949 & $34.105(1.00)$ & 35.712 & $34.522(0.70)$ & 36.531 & $33.954(0.80)$ & 36.314 \\
\hline
\end{tabular}

The number in parentheses represents the standard deviation

Isotropic skew plates

The thickness of the isotropic skew plates was taken as $2.0 \mathrm{~mm}$. The aspect ratio was varied from 1.0 to 2.5 and the skew angle from $0^{\circ}$ to $45^{\circ}$. Table 1 and Fig. 2 show the variation of non-dimensional frequency coefficient $K_{\mathrm{f}}$ with aspect ratio and skew angle for isotropic skew plates. The variation of first natural frequency with an increase in the aspect ratio is not appreciable up to a skew angle of about $30^{\circ}$. The variation of second and third natural frequencies with an increase in the aspect ratio for a given skew angle is considerable. The first, second and third natural frequencies increase monotonically with an increase in the skew angle for any given value of aspect ratio. The experimental values of the non-dimensional frequency coefficient $K_{\mathrm{f}}$ are in very good agreement with those of the finite element solution. The first three mode shapes obtained by finite element analysis are shown in Fig. 3 for aspect ratio = 1.0. 
Table 5 Non-dimensional frequency coefficient for laminated composite skew plates $\left(\alpha=45^{\circ}\right)$

\begin{tabular}{|c|c|c|c|c|c|c|c|c|c|}
\hline \multirow{4}{*}{$\begin{array}{l}\text { Laminate staking } \\
\text { sequence }\end{array}$} & \multirow{4}{*}{$\begin{array}{l}\text { Mode } \\
\text { number }\end{array}$} & \multicolumn{8}{|c|}{ Non-dimensional frequency coefficient $\left(K_{f}\right)$} \\
\hline & & \multicolumn{8}{|c|}{ Aspect ratio $(a / b)$} \\
\hline & & \multicolumn{2}{|l|}{1.0} & \multicolumn{2}{|l|}{1.5} & \multicolumn{2}{|l|}{2.0} & \multicolumn{2}{|l|}{2.5} \\
\hline & & Experiment & FEM & Experiment & FEM & Experiment & FEM & Experiment & FEM \\
\hline \multirow{3}{*}{$\begin{array}{l}\text { Angle-ply } \\
\qquad\left[+0^{\circ} /-0^{\circ} \% / 0^{\circ}\right]\end{array}$} & 1 & $18.149(0.20)$ & 18.711 & $17.719(0.19)$ & 18.457 & $17.593(0.22)$ & 18.137 & $17.515(0.11)$ & 17.872 \\
\hline & 2 & $19.284(0.28)$ & 20.087 & $20.491(0.22)$ & 21.570 & $21.794(0.45)$ & 23.186 & $23.288(0.67)$ & 25.040 \\
\hline & 3 & $26.964(0.50)$ & 28.087 & $37.360(0.80)$ & 39.327 & $46.893(2.00)$ & 49.887 & $46.186(1.85)$ & 49.662 \\
\hline \multirow{3}{*}{$\begin{array}{l}\text { Angle-ply } \\
\qquad\left[+45^{\circ} /-45^{\circ} / \ldots /-45^{\circ}\right]\end{array}$} & 1 & $17.949(0.22)$ & 18.697 & $16.312(0.15)$ & 17.171 & $14.077(0.33)$ & 14.975 & $12.561(0.34)$ & 13.506 \\
\hline & 2 & $18.365(0.31)$ & 19.130 & $19.842(0.25)$ & 20.886 & $23.149(0.45)$ & 24.627 & $26.238(0.80)$ & 28.213 \\
\hline & 3 & $28.674(0.54)$ & 29.869 & $37.477(1.25)$ & 39.449 & $43.781(1.29)$ & 46.576 & $40.812(1.56)$ & 43.884 \\
\hline \multirow{3}{*}{$\begin{array}{l}\text { Angle-ply } \\
\qquad\left[+90^{\circ} /-90^{\circ} / \ldots /-90^{\circ}\right]\end{array}$} & 1 & $13.011(0.16)$ & 13.554 & $11.513(0.12)$ & 12.119 & $10.253(0.13)$ & 10.907 & $9.411(0.15)$ & 10.119 \\
\hline & 2 & $14.626(0.13)$ & 15.235 & $15.792(0.14)$ & 16.623 & $17.033(0.35)$ & 18.121 & $18.577(0.40)$ & 19.976 \\
\hline & 3 & $25.705(0.60)$ & 26.776 & $30.856(1.10)$ & 32.480 & $29.319(0.90)$ & 31.190 & $27.562(0.77)$ & 29.636 \\
\hline \multirow{3}{*}{$\begin{array}{l}\text { Cross-ply } \\
{\left[0^{\circ} / 90^{\circ} \% / . .190^{\circ}\right]}\end{array}$} & 1 & $16.062(0.17)$ & 16.731 & $15.304(0.25)$ & 16.109 & $14.547(0.32)$ & 15.475 & $13.928(0.33)$ & 14.976 \\
\hline & 2 & $17.989(0.18)$ & 18.739 & $19.401(0.32)$ & 20.422 & $20.551(0.40)$ & 21.863 & $21.952(0.50)$ & 23.604 \\
\hline & 3 & $29.705(0.55)$ & 30.943 & $39.355(0.75)$ & 41.427 & $40.157(0.95)$ & 42.721 & 38.893 (1.60) & 41.820 \\
\hline
\end{tabular}

The number in parentheses represents the standard deviation

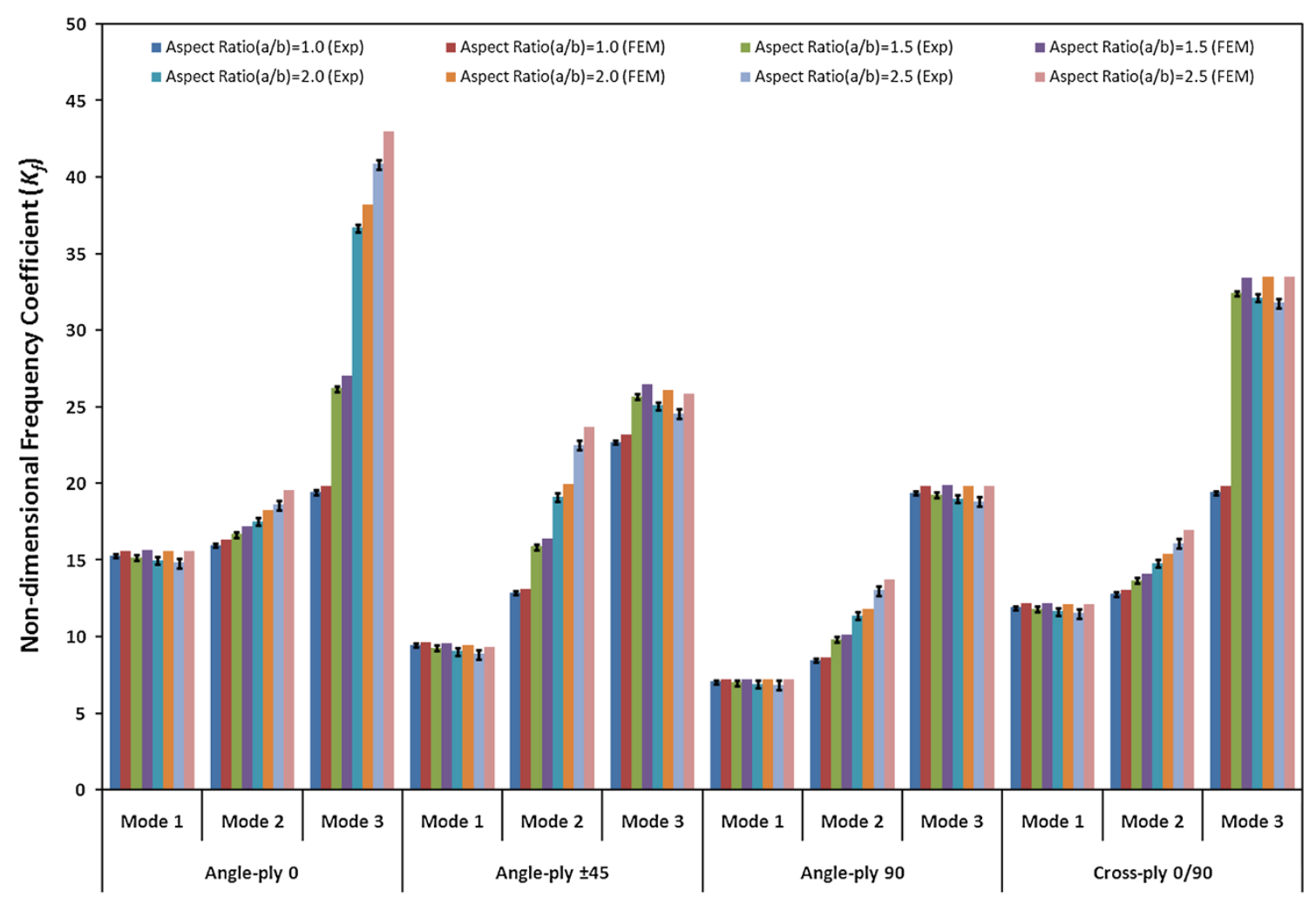

Antisymmetric Laminate Staking Sequence

Fig. 4 Variation of $K_{\mathrm{f}}$ with aspect ratio $(a / b)$ for different laminate stacking sequences $\left(\alpha=0^{\circ}\right)$ 


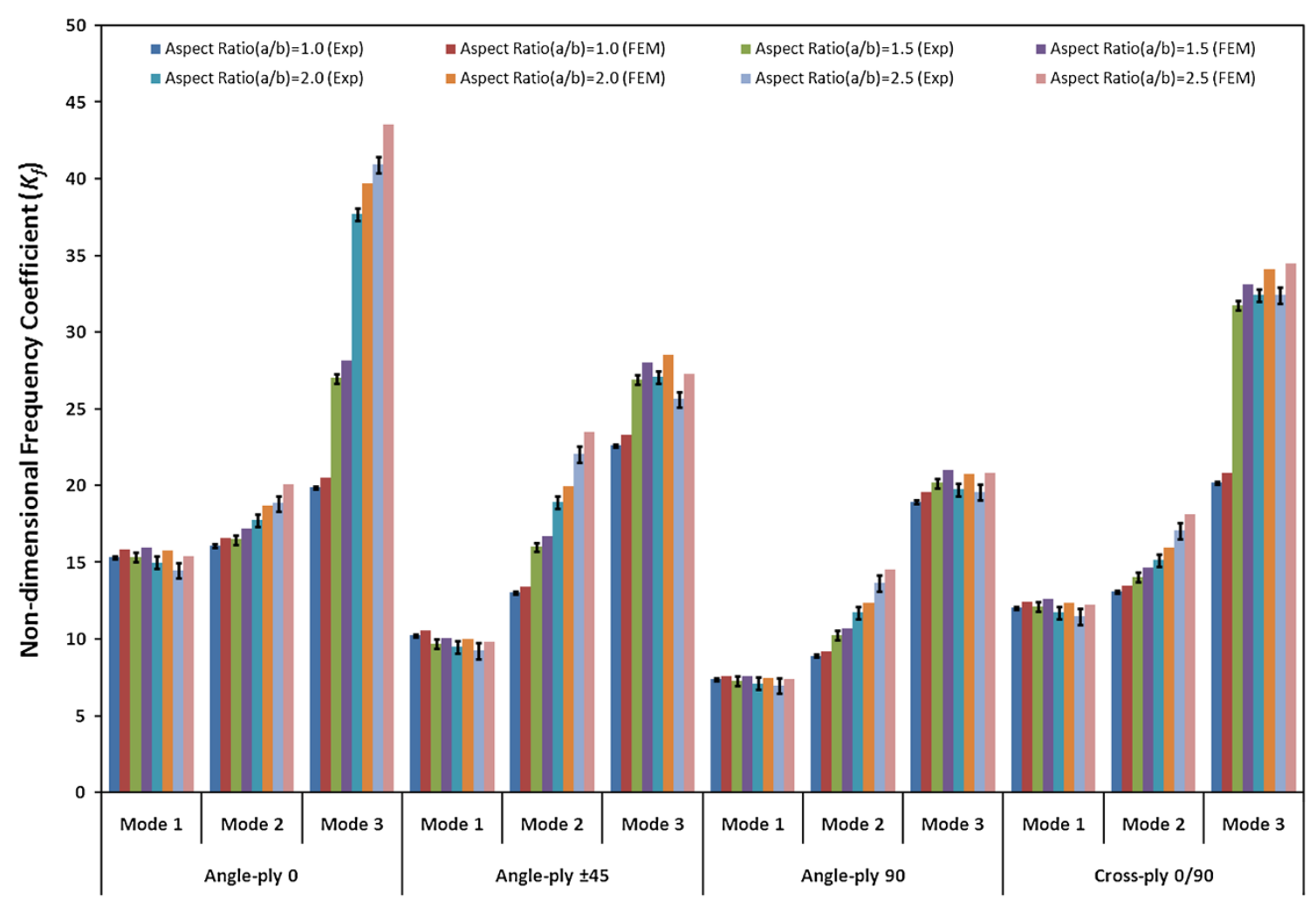

Antisymmetric Laminate Staking Sequence

Fig. 5 Variation of $K_{\mathrm{f}}$ with aspect ratio $(a / b)$ for different laminate stacking sequences $\left(\alpha=15^{\circ}\right)$

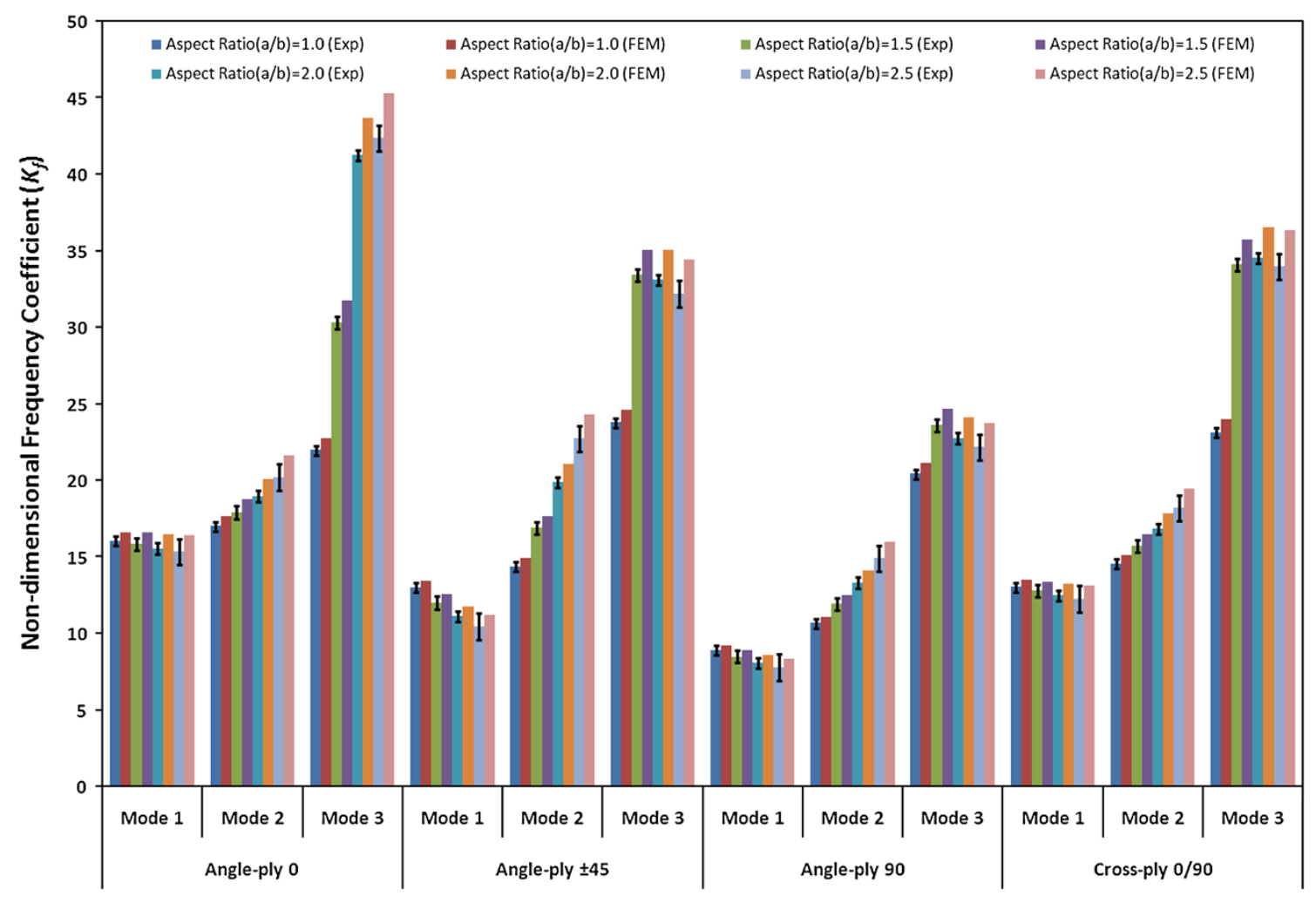

Antisymmetric Laminate Staking Sequence

Fig. 6 Variation of $K_{\mathrm{f}}$ with aspect ratio $(a / b)$ for different laminate stacking sequences $\left(\alpha=30^{\circ}\right)$ 


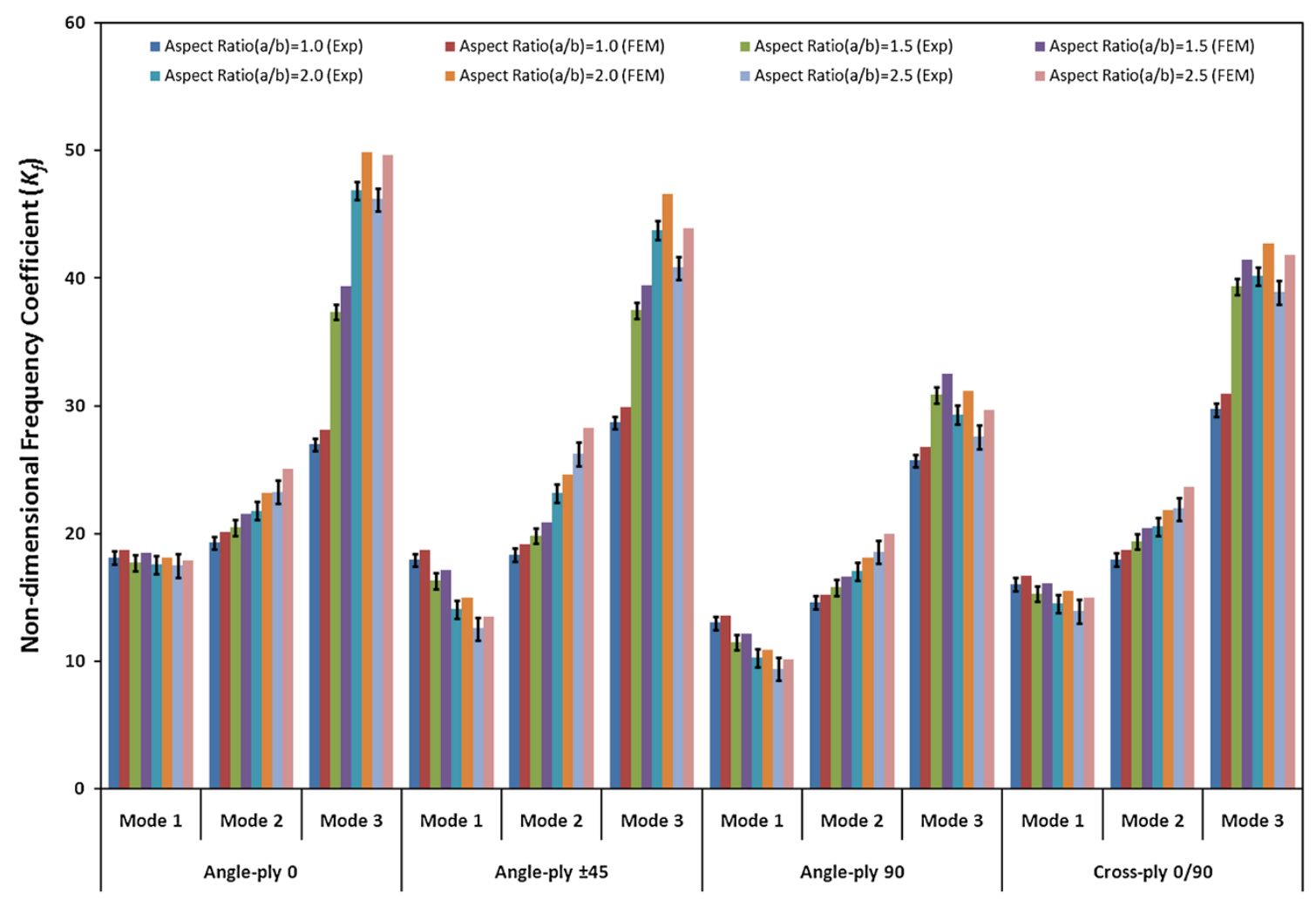

Antisymmetric Laminate Staking Sequence

Fig. 7 Variation of $K_{\mathrm{f}}$ with aspect ratio $(a / b)$ for different laminate stacking sequences $\left(\alpha=45^{\circ}\right)$

Laminated composite skew plates

The total thickness of the laminate was maintained constant at $2 \mathrm{~mm}$, the number of layers being 20 . The aspect ratio was varied from 1.0 to 2.5 and the skew angle from $0^{\circ}$ to $45^{\circ}$. Tables 2, 3, 4, 5 and Figs. 4, 5, 6, 7 show the variation of non-dimensional frequency coefficient $K_{\mathrm{f}}$ with aspect ratio and laminate stacking sequence for various values of skew angle. Four laminate stacking sequences viz., antisymmetric angle-ply $\left[+0^{\circ} /-0^{\circ} \% /-0^{\circ}\right]$, antisymmetric angle-ply $\left[+45^{\circ} /-45^{\circ} \% /-45^{\circ}\right]$, antisymmetric angle-ply $\left[+90 \%-90^{\circ} \% /-90^{\circ}\right]$ and antisymmetric cross-ply $\left[0^{\circ} / 90^{\circ} \%\right.$ ... $\left./ 90^{\circ}\right]$ were considered. It is seen that the first natural frequency decreases with an increase in the aspect ratio for all laminate stacking sequences. The second natural frequency increases with an increase in the aspect ratio for all laminate stacking sequences. For laminate stacking sequence $\left[+0^{\circ} /-0^{\circ} \% /-0^{\circ}\right]$, the third natural frequency increases with an increase in the aspect ratio except in the case of skew angle $=45^{\circ}$ where it increases up to aspect ratio $=2.0$ and later decreases. For laminate stacking sequence $\left[+45^{\circ} /-45^{\circ} \% /-45^{\circ}\right]$, the third natural frequency first increases and then decreases with an increase in the aspect ratio for all skew angles. For laminate stacking sequence $\left[+90^{\circ} /-90^{\circ} \% \ldots /-90^{\circ}\right]$, the third natural frequency decreases monotonically with an increase in the aspect ratio for skew angle $=0$ whereas for other skew angles, it first increases and then decreases. For laminate stacking sequence $\left[0^{\circ} / 90^{\circ} \% / . . / 90^{\circ}\right]$, the third natural frequency first increases and later decreases with an increase in the aspect ratio for all the skew angles considered. Other parameters being the same, the antisymmetric angle-ply $\left[+0^{\circ} \%-0 \%\right.$ .../ $\left.-0^{\circ}\right]$ yields the highest value for the first natural frequency and the antisymmetric angle-ply $[+90 \%-90 \%$. $90^{\circ}$ ] yields the lowest value. The first natural frequency for the other laminate stacking sequences lies in between the above two extreme values. The first, second and third natural frequencies generally increase with an increase in the skew angle for any given value of aspect ratio for all the laminate stacking sequences. The first three mode shapes obtained by finite element analysis are shown in Fig. 8 for antisymmetric cross-ply $\left[0^{\circ} / 90^{\circ} / \ldots / 90^{\circ}\right]$ for aspect ratio $=1.0$. The natural frequency depends on the stiffness of the cross-section of the laminate among other factors such as boundary conditions etc. The stiffness of the crosssection depends upon the contribution made by extensional stiffness, coupling stiffness and bending stiffness terms (Jones 1975). 


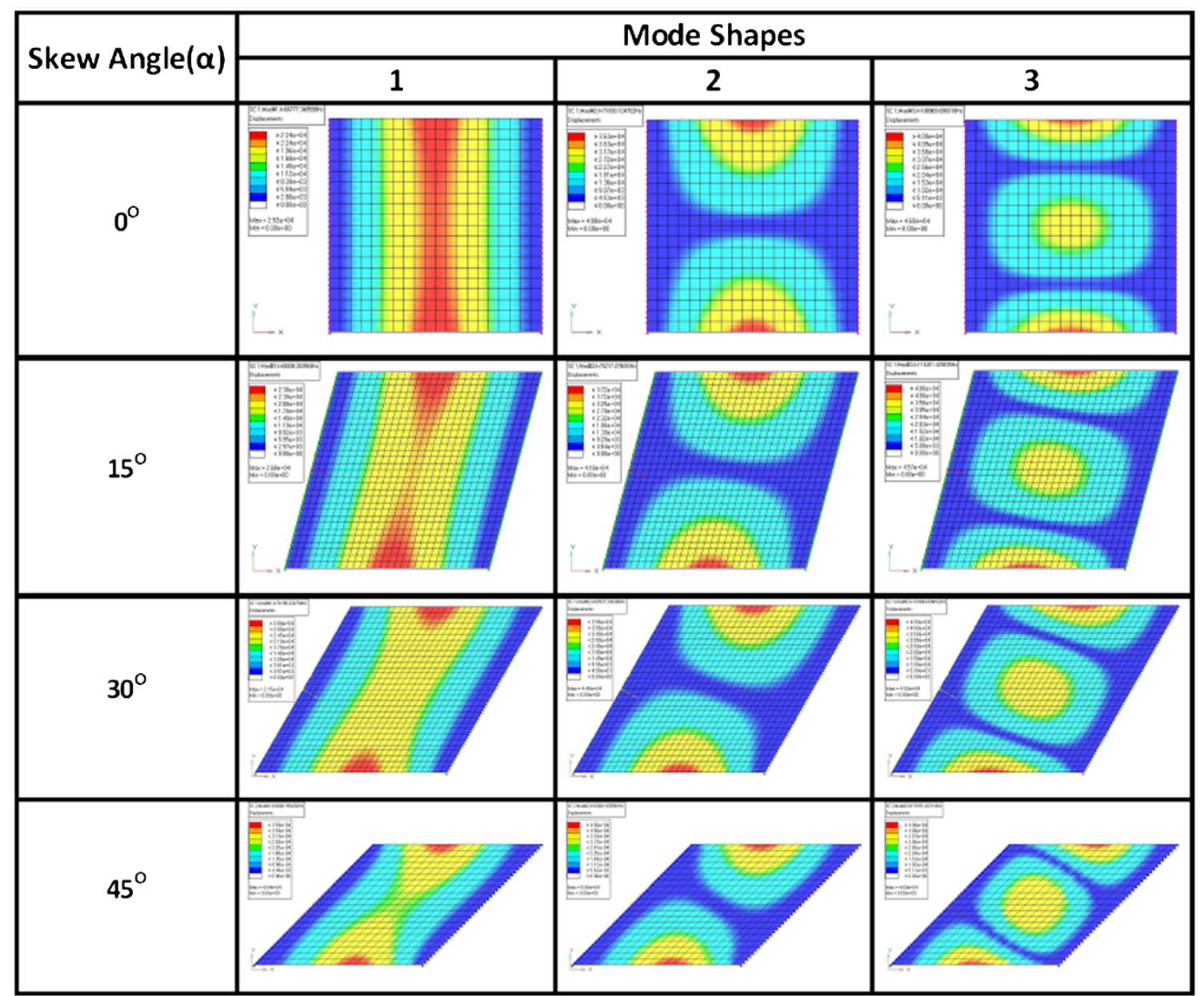

Fig. 8 Mode shapes for laminated composite skew plates $(a / b=1.0, \mathrm{NL}=20$, antisymmetric cross-ply laminate)

\section{Conclusions}

The following conclusions are made based on the above study.

- The experimental values of the first, second and third natural frequencies are in good agreement with those of the finite element solution in the case of both isotropic and laminated composite skew plates.

- In the case of isotropic skew plates, the variation of first natural frequency with an increase in the aspect ratio is not appreciable up to a skew angle of about $30^{\circ}$. The second natural frequency increases with an increase in the aspect ratio for all laminate stacking sequences. The first, second and third natural frequencies increase monotonically with an increase in the skew angle for any given value of aspect ratio.

- In the case of laminated composite skew plates, the first natural frequency decreases with an increase in the aspect ratio for all laminate stacking sequences. The variation of second and third natural frequencies with aspect ratio is considerable in the range of skew angle considered. Other parameters being the same, the antisymmetric angle-ply $\left[+0 \%-0 \% \ldots /-0^{\circ}\right]$ yields the highest value for the first natural frequency and the antisymmetric angle-ply $\left[+90^{\circ} /-90^{\circ} \% /-90^{\circ}\right]$ yields the lowest value. The first natural frequencies for the other laminate stacking sequences lie in between these two extreme values. The first, second and third natural frequencies generally increase with an increase in the skew angle for any given value of aspect ratio.

Acknowledgments The first author would like to thank the Management and Principal Dr. S.G. Hiremath of GM Institute of Technology, Davangere, Karnataka, India for the kind encouragement and support provided. The second author would like to thank the Management of Jawaharlal Nehru College of Engineering, Shivamogga, Karnataka, India for the kind encouragement and support provided. The third author would like to thank the Management, Principal Dr. N Ranaprathap Reddy and Head of the Department of Civil Engineering Dr. Y. Ramalinga Reddy, Reva Institute of Technology and Management, Bangalore, Karnataka, India for the kind encouragement and support provided.

Open Access This article is distributed under the terms of the Creative Commons Attribution License which permits any use, 
distribution, and reproduction in any medium, provided the original author(s) and the source are credited.

\section{References}

ASTM D 3039/D 3039M (2006) Standard test method for tensile properties of polymer matrix composite materials

Bardell NS (1992) The free vibration of skew plates using the hierarchical finite element method. Comput Struct 45:841-874

Barton MV (1951) Vibration of rectangular and skew cantilever plates. J Appl Mech ASME 18:129-134

Cawley P, Adams RD (1978) The predicted and experimental natural modes of free-free CFRP plates. J Compos Mater 12:336-347

Chakraborty S, Mukhopadhyay M, Mohanty AR (2000) Free vibrational responses of FRP composite plates: experimental and numerical studies. JRPC 19:535-551

Clary RL (1975) Vibration characteristics of aluminium plates reinforced with boron-epoxy composite material. J Compos Mater 7:348-365

Classen RW (1963) Vibration of skew cantilever plates. AIAA J 1:1222-1228

Conway HD, Farnham KA (1965) The free flexural vibrations of triangular rhombic and parallelogram plates and some analogies. Int J Mech Sci 7:811-816

Durvasula S (1969) Natural frequencies and modes of clamped skew plates. AIAA J 7:1164-1166

Dutt KM, Shivanand HK (2011) An experimental approach to free vibration response of carbon composite laminates. J Adv Eng Appl 66-68

Garg AK, Khare RK, Kant T (2006) Free vibration of skew fiberreinforced composite and sandwich laminates using a shear deformable finite element model. J Sandw Struct Mater 8:33-53

Hamada M (1959) Compressive or shearing buckling load and fundamental frequency of a rhomboidal plate with all edges are clamped. Bull JSME 2:520-526

Hasegawa M (1957) Vibration of clamped parallelogrammic isotropic flat plates. J Aeronaut Sci 24:145-146

Jones RM (1975) Mechanics of composite materials. McGraw-Hill, New York

Kamal K, Durvasula S (1986) Some studies on free vibration of composite laminates. Compos Struct 5:177-202

Kaul RM, Cadambe V (1956) The natural frequencies of thin skew plates. Aeronaut Q 7:37-352

Krishna Reedy AR, Palaninathan R (1999) Free vibration of skew laminates. Comput Struct 70:415-423

Krishnan A, Deshpande JV (1992) Vibration of skew laminates. J Sound Vib 153(2):351-358

Kuttler JR, Sigillito VG (1980) Upper and lower bounds for frequencies of clamped rhombical plates. J Sound Vib 68:597-607

Laura PA, Grosson J (1968) Fundamental frequency of vibration of rhombic plates. ASAJ 44:823-824

Liew KM, Lam KY (1990) Application of two-dimensional orthogonal plate functions to flexural vibration of skew plates. J Sound Vib 132(2):241-252

Liew KM, Wang CM (1993) Vibration studies on skew plates: treatment of internal line supports. Comput Struct 49(6):941-951

Malhotra SK, Ganesan N, Veluswami MA (1988) Effect of fiber orientation and boundary conditions on the vibration behavior of orthotropic rhombic plates. J Compos Struct 19(2):127-132

Maruyama K, Ichinomiya O, Narita Y (1983) Experimental study of the free vibration of clamped trapezoidal plates. J Sound Vib 88(4):523-534

McGee OG, Butalia TS (1994) Natural frequencies of shear deformable cantilevered skew thick plates. J Sound Vib 176:351-376
Mizusawa T, Kajita T (1986) Vibration and buckling of skew plates with edges elastically restrained against rotation. Comput Struct 22:987-994

Mizusawa T, Kajita T (1987) Vibration of skew plates resting on point supports. J Sound Vib 15:243-251

Mizusawa T, Kajita T, Naruoka M (1979) Vibration of skew plates by using b-spline functions. J Sound Vib 62:301-308

Mizusawa T, Kajita T, Naruoka M (1980) Analysis of skew plate problems with various constraints. J Sound Vib 68:575-584

Monforton GR (1968) Finite element displacement and vibration analysis of skew plates, Report 18. Structural and Mechanical Western Reserve University, Cleveland

Nair PS, Durvasula S (1973) Vibration of skew plates. J Sound Vib 26(1):1-19

Singh B, Chakraverthy S (1994) Flexural vibration of skew plates using boundary characteristic orthogonal polynomials in two variables. J Sound Vib 173:157-178

Singha MK, Ganapathi M (2004) Large amplitude free flexural vibrations of laminated skew plates. Int $\mathrm{J}$ Nonlinear Mech 39:1709-1720

Srinivasa CV, Suresh YJ, Prema Kumar WP (2012) Free flexural vibration studies on laminated composite skew plates. IJEST 4(4):13-24

Srinivasan RS, Ramachandran SV (1975) Vibration of generally orthotropic skew plates. J Acoust Soc Am 57:1113-1118

Thangam Babu PV, Reddy DV (1971) Frequency analysis of skew orthotropic plates by the finite strip method. J Sound Vib 18(4):465-474

C. V. Srinivasa received M. Tech. from Visvesvaraya Technological University, Belgaum, India in 2003 by securing III Rank. He is an Assistant Professor in the Department of Mechanical Engineering, GM Institute of Technology, Davangere, Karnataka, India-577006. Presently, he is pursuing Ph.D at Research Centre, Department of Mechanical Engineering, J.N.N. College of Engineering, Shivamogga, affiliated to Visvesvaraya Technological University, Karnataka, India. His research interests include stress analysis, finite element method and experimental material characterization. $\mathrm{He}$ is a Member of ISTE (India), ISTAM.MRSI.

Y. J. Suresh is a Professor in the Department of Mechanical Engineering, J.N.N. College of Engineering, Shivamogga, Karnataka, India. He received Ph.D. from Indian Institute of Technology, Madras, India. He has more than 30 years of experience in teaching and research. His current area of research includes Design, Stress analysis, finite element analysis, vibration. He has published many papers in referred international journals. He is a Fellow of IE (India), ISTE (India), SEM, AMAeI (Associate Member of Aeronautical Society of India).

W. P. Prema Kumar is a Senior Professor in the Department of Civil Engineering, Reva Institute of Technology and Management, Bangalore, Karnataka, India-560064, India. He is also a Member of Academic Council, Reva University, Bangalore, Karnataka, India560064. He received Ph.D. from Indian Institute of Technology, Madras, India. He has more than 35 years of experience in teaching and research. His current area of research includes Finite Element Analysis and Laminated Composite Structures. He has published many papers in referred national and international journals. He is a Member of IE (India), ISTE (India), American Society of Civil Engineers (India Section) and Member of Indian Standards. 